\title{
Modeling circumstellar envelope with advanced numerical codes
}

\author{
P. Procopio ${ }^{1}$, A. De Rosa ${ }^{1}$, C. Burigana ${ }^{1}$, \\ G. Umana ${ }^{2}$, and C. Trigilio ${ }^{2}$ \\ ${ }^{1}$ INAF - IASF Bologna, Via P. Gobetti 101, 40129 Bologna \\ ${ }^{2}$ INAF - OACt, Via S. Sofia 78, Catania
}

\begin{abstract}
We propose a modeling study on the formation and evolution of the Circumstellar Envelopes (CSEs) of a sample of selected radio-loud objects, based on an innovative interaction between two codes widely used by the scientific community, but in different fields. CLOUDY $\dagger$ (Ferland et al. 1998) is a widely used code to model the spectral energy distribution (SED) of the several objects characterized by clouds of gas heated and ionized by a central object. CosmoMC $\ddagger$ (Lewis \& Bridle 2002) instead is usually used for exploring cosmological parameter space. We investigate here on the exploitation of the sampling performance of the Markov-Chain MonteCarlo (MCMC) engine of CosmoMC to search for a best fit model of the considered objects through the spectral synthesis capacity of CLOUDY.
\end{abstract}

Keywords. circumstellar matter, planetary nebulae, MCMC

\section{Introduction}

Detailed observations of (CSE) are of crucial importance in determining fine details of different aspects related to the physical properties of plasma and dust around various classes of stars in their late evolution stages. The modeling and characterization of the CSEs are of extreme relevance for understanding the formation and evolution of relatively fast evolving objects, like e.g., Planetary Nebulae (PN), on which this contribution is focused. On the other side, the enrichment of the Interstellar Medium (ISM) depends on the chemistry and the composition of gas and dust that are coming out from PNs and Supernovae, through stellar winds or shockwaves. In this study, the spectral emission is predicted through CLOUDY, a performing public $\mathrm{C}++$ based code, while the core of the optimization of the model parameters is CosmoMC, a FORTRAN90 MCMC engine, supplied with additional codes to analyze specific output data of the sampler.

\section{The Data-set}

The sample of PNs has been extracted from the Condon \& Kaplan catalogue (1998). Only objects characterized by a flux density higher than $100 \mathrm{mJy}$ at $1.4 \mathrm{GHz}$ were selected (in order to guarantee a good detection with the Noto radiotelescope). Some observations has been already performed at 8.4 and $43 \mathrm{GHz}$ (Umana et al. 2008). To consider the dust emission associated to the PNs, data from near $(2 \mu \mathrm{m})$ to far $(100 \mu \mathrm{m})$ infrared were retrieved as well: photometric measurements have been extracted from IRAS, 2MASS, and MSX archives.

$\dagger$ CLOUDY is available for free download at http://www.nublado.org $\ddagger$ ComoMC is available for free download at http://cosmologist.info/cosmomc/ 


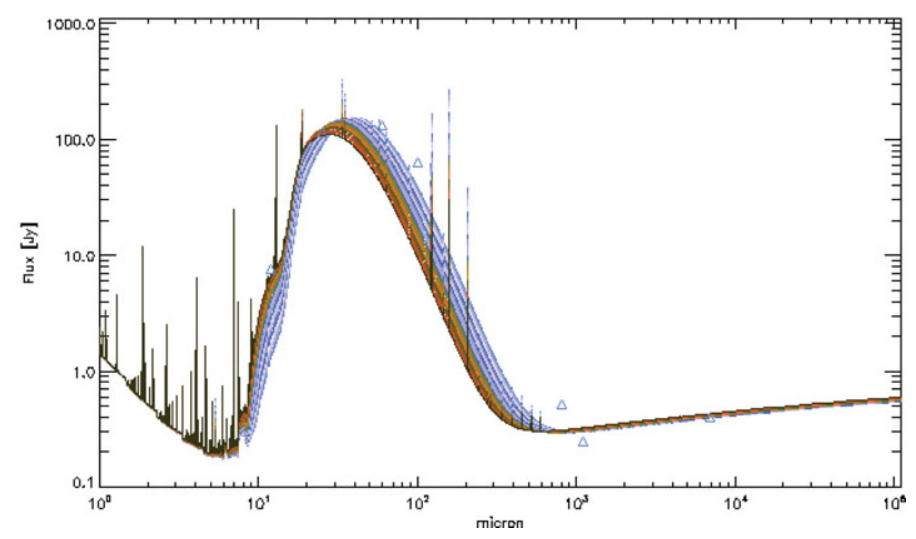

Figure 1. The effect of the variation of the density parameter of the SED model for NGC6543: increasing the parameter the peak of the model shifts to higher frequencies. The observed data are indicated with blue triangles.

\section{Modeling through CLOUDY}

We make wide use of the spectral synthesis code CLOUDY in order to fit models to observational data. We assume a reasonable set of initial parameters (central star radiation field, CSE density distribution, elements abundances and properties, geometric structure, filling factor, distance of the PN) describing the ionizing star of the considered $\mathrm{PN}$. Once the initial model is obtained, it is possible to perform a fine tuning of some critical parameters in order to achieve their best estimate and to derive a fiducial model for the considered object. The variation of the parameters could be done inside CLOUDY itself, through its own optimization method, but it is necessary to provide a good initial guess of the parameters.

Astrophysical parameters - We focus in particular on the parameters essential for the modelization of the observed SED. Among them, we remember: the inner radius and the extension of the ionized plasma region, with typical values, for the inner radius, that may span between $10^{16}$ and $10^{19} \mathrm{~cm}$; the filling factor, that takes into account the small scale structure of the nebula; the temperature of the central star, that may range from about ten to many tens of thousands Kelvin, and the total density of $\mathrm{H}\left(\mathrm{HII}+\mathrm{HI}+\mathrm{H}_{2}\right)$ in the $\mathrm{PN}$, that may range from some thousands to a million of atoms per $\mathrm{cm}^{3}$. Also, the dust composition (astronomical silicate and/or graphite) and the grain dimensions play a fundamental role in shaping of the SED. These parameters act differently on the SED, but a certain degree of degeneracy persists.

Optimization process - The optimization process in CLOUDY is performed by the PHYMIR algorithm (van Hoof 1997), a non-standard fit procedure for minimizing the $\chi^{2}$. The observables are divided in four categories: emission lines ratio, infrared emission, radio flux or $\mathrm{H}-\beta$ line, and angular diameter. The total $\chi^{2}$ of the model is computed considering the contribution of each observable: $\chi_{i}^{2}=\left[\left(O_{i}^{m}-O_{i}^{o}\right) /\left(\min \left(O_{i}^{m}, O_{i}^{o}\right) \sigma_{i}\right)\right]^{2}$. Here the suffix $o$ identifies the observed quantity, while $m$ the value obtained through the model for the considered observable, $\sigma$ the error on the observed quantity $i$. Anyway, some problems can be encountered: possibility of a non-converging optimization phase; long lasting runs; errors due to chance of convergence to a secondary minimum of the $\chi^{2}$. We develop an alternative method for the optimization of the models exploiting the power of the MCMC sampling, having also the possibility to test different minimization criteria. 


\section{The Markov-Chain Monte-Carlo sampler}

The extraction of the parameters needed to run CLOUDY is performed through the code CosmoMC. Being originally designed for cosmological applications, CosmoMC can also be compiled as a generic sampler, providing a likelihood function, through which behavior, the sampler drives the extractions in order minimize the differences between models and observations.

\section{Interface between the codes}

Unfortunately, making the codes talking is not that simple. The principal difficulty lies in the different programming language of the codes. The parameters exchange between the codes is then a crucial phase and several modifications have to be made on CosmoMC. To set up properly the sampler it is necessary to define and introduce the new parameters in more than one file of the CosmoMC package. Through the MCMC sampler it is possible to work in two ways: $i$ ) finding the best fit of the fiducial model; $i i$ ) probing the entire space defined by the parameters one wants to recover. The first option is the faster, but no additional informations are available on the sampling. The second is of course slower, but allows one to deeply analyze the extraction history, checking, for example, for secondary minima in the distribution of the models.

\section{Discussion and conclusion}

The computational time depends strongly on the required number of iterations, on the choice of the sampling method, and on the number of chains in the sampling: for about twenty objects, considering about $3 \times 10^{4}$ iterations and 8 chains, the computational time estimate is around $2 \times 10^{5} \mathrm{CPU}$ hours, in line with computational resources available on new generation of extreme multi-core platforms. Our first tests indicate that the fiducial model recovered through the interactions of these two codes, could be more solid. In addition, a complete scan of the parameter space can be performed, allowing to accurately analyze the behavior of each crucial parameter. Furthermore, an additional tuning could come from a proper definition of the likelihood function.

\section{Acknowledgement}

We acknowledge the use of the computational facilities of INAF-IASF Bologna. We acknowledge partial support by the ASI/INAF Agreement I/072/09/0 for the Planck LFI Activity of Phase E2 and the ASI contract I/016/07/0 COFIS. We acknowledge the use of the CosmoMC code Lewis \& Bridle 2002. Calculations were performed with version 08.00 of CLOUDY, last described by Ferland et al. 1998.

\section{References}

Condon, J. J. \& Kaplan, D. L. 1998, ApJ Supp. Series, 117, 361

Ferland, G. J., Korista, K. T., Verner, D. A., Ferguson, J. W., Kingdon, J. B., \& Verner, E. M. 1998, PASP, 110, 761

Lewis, A. \& Bridle, S. 2002, Phys. Rev. D, 66, 103511

Umana, G., Leto, P., Trigilio, C., Buemi, C. S., Manzitto, P., Toscano, S., Dolei, S., \& Cerrigone, L. 2008, A\&A, 482, 529

van Hoof, P., PhD Thesis, http://homepage.oma.be/pvh/thesis.html 